\title{
Augmented pulmonary IL-4 and IL-13 receptor subunit expression in idiopathic interstitial pneumonia
}

\author{
C Jakubzick, E S Choi, S L Kunkel, H Evanoff, F J Martinez, R K Puri, K R Flaherty, G B Toews, \\ T V Colby, E A Kazerooni, B H Gross, W D Travis, C M Hogaboam
}

J Clin Pathol 2004;57:477-486. doi: 10.1136/jcp.2003.012799

See end of article for authors' affiliations

Correspondence to: Dr C M Hogaboam, Department of Pathology, University of Michigan Medical School, Room 5214, Med Sci I, 1301 Catherine Road, Ann Arbor Ml 48109-0602, USA; Hogaboam@med. umich.edu

Accepted for publication 19 November 2003
Background: Some idiopathic interstitial pneumonias (IIPs) are characterised by fibroproliferation and deposition of extracellular matrix. Because efficacious treatment options are limited, research has been directed towards understanding the cytokine networks that may affect fibroblast activation and, hence, the progression of certain IIPs.

Aims: To examine the expression of interleukin 4 (IL-4), IL-13, and their corresponding receptor subunits in the various forms of IIP and normal patient groups.

Methods: Molecular and immunohistochemical analysis of IL-4, interferon $\gamma$ (IFN $\gamma$ ), IL-13, IL-4 receptor (IL-R), and IL-13 receptor subunits in surgical lung biopsies (SLBs) from 39 patients (21 usual interstitial pneumonia (UIP), six non-specific interstitial pneumonia (NSIP), eight respiratory bronchiolitic interstitial lung disease (RBILD), and five normal controls).

Results: Molecular analysis demonstrated that IL-13R $\alpha 2$, IL-13R $\alpha 1$, and IL-4R $\alpha$ were present in a greater proportion of upper and lower lobe biopsies from patients with UIP than patients with NSIP and RBILD. Immunohistochemical analysis of patients with UIP, NSIP, and RBILD revealed interstitial staining for all three receptor subunits, whereas such staining was only seen in mononuclear cells present in normal SLBs. Fibroblastic foci in patients with UIP strongly stained for IL-4R $\alpha$ and IL-13R $\alpha 2$. Localised expression of IL-4R $\alpha$ was also seen in SLBs from patients with NSIP but not in other groups.

Conclusion: Some histological subtypes of IIP are associated with increased pulmonary expression of receptor subunits responsive to IL-4 and IL-13. These findings may be of particular importance in understanding the pathogenesis of IIP and, more importantly, may provide important novel therapeutic targets. hronic pulmonary fibrosis of known and idiopathic origin presents extraordinary clinical challenges for which treatment options show limited effectiveness ${ }^{1}$ or considerable toxicity, ${ }^{2}$ and the median survival rate following diagnosis has changed little. ${ }^{34}$ Idiopathic interstitial pneumonia (IIP) encompasses a diverse group of disorders involving the distal pulmonary parenchyma, which share numerous features, but are felt to be sufficiently different to justify designation as separate disorders..$^{5-8}$ The pathogenesis of IIP is unclear, although it is thought to centre around an injury to the lung, followed by attempts to heal this injury. Fibroblastic foci, small aggregates of actively proliferating fibroblasts, are thought to represent previous foci of injury and indicate that fibrosis is active and ongoing. Recent studies have renewed interest in categorising patients with IIP according to histological features. ${ }^{7-10}$ Patients with IIP are divided into numerous histological categories, including usual interstitial pneumonia (UIP), non-specific interstitial pneumonia (NSIP), and respiratory bronchiolitic interstitial lung disease (RBILD). The presence of fibroblastic foci is a key histological feature in the differential diagnosis. ${ }^{7}$ Patients with NSIP have a better prognosis and response to immunosuppressive treatment than those with UIP. ${ }^{11-13}$ In addition, those patients with a predominantly cellular form of NSIP appear to have a better prognosis than those showing a predominantly fibrotic form of NSIP. ${ }^{713}$

Several studies have suggested that chronic IIP, particularly UIP, is characterised by a skewed cytokine pattern. For example, surgical lung biopsies (SLBs) from patients with UIP exhibit a predominance of T helper type 2 (Th2) cytokine (interleukin 4 (IL-4) and IL-13) over Thl cytokine (interferon $\gamma($ IFN $\gamma)$ and IL-12) gene and protein expression compared with similar biopsies from patients with non-fibrotic disease ${ }^{14}{ }^{15}$ sarcoid, ${ }^{16}$ and systemic sclerosis. ${ }^{17}$ IL-4 has been shown to be a potent inducer of transforming growth factor $\beta$ production by pulmonary fibroblasts, leading to enhanced type I and III collagen, fibronectin, and tenascin synthesis. ${ }^{18-21}$ IL-4 has also been shown to be a potent chemotactic factor for the directed movement of pulmonary fibroblasts. ${ }^{22}$ IL-13 also exerts major profibrotic effects in the lung. ${ }^{23-27}$ The profibrotic effect of IL-13 in the lung appears to involve irreversible fibroblast activation, triggered either directly $y^{28}$ or indirectly through transforming growth factor $\beta .^{24}{ }^{30} \mathrm{~A}$ recently published Affymetrix gene array analysis of human lung fibroblasts showed that IL-13 has major stimulatory effects on a multitude of genes that contribute to lung remodelling or fibrotic events. ${ }^{31}$ More recently, Homer and colleagues $^{32}$ postulated from their studies of IL-13 transgenic mice that IL-13 may play a central role in the broad range of chronic pulmonary conditions in which fibrosis, type II cell hypertrophy, and surfactant accumulation occur. Furthermore, IL-13 is pertinent to IIP, given that concentrations of

Abbreviations: ELISA, enzyme linked immunosorbent assay; FF, fibroblastic foci profusion; GAPDH, glyceraldehyde 3-phosphate dehydrogenase; $\mathrm{HRCT}$, high resolution computed tomography; IFN $\gamma$, interferon $\gamma$; IIP, idiopathic interstitial pneumonia; IL, interleukin; IL- $4 R$, IL-4 receptor; IRAP, interleukin 1 receptor antagonist protein; NSIP, nonspecific interstitial pneumonia; RBILD, respiratory bronchiolitic interstitial lung disease; RT-PCR, reverse transcription polymerase chain reaction; SLB, surgical lung biopsy; Th1, T helper type 1; UIP, usual interstitial pneumonia 
this cytokine are greatly upregulated in alveolar macrophages from patients with IIP. ${ }^{33}$

\section{"Several studies have suggested that chronic idiopathic interstitial pneumonia, particularly usual interstitial pneu- monia, is characterised by a skewed cytokine pattern"}

At present, little is known about the expression of the receptor subunits that bind IL-4 and IL-13 in the context of IIP. IL-4 directly and exclusively binds the IL- 4 receptor $\alpha$ (IL$4 \mathrm{R} \alpha$ ) chain linked to the common $\gamma$ chain. The common $\gamma$ chain also forms an important functional component of the IL-2, IL-7, IL-9, IL-15, and IL-21 receptors. ${ }^{34}$ IL- 13 specifically binds the IL-13R $\alpha$ l chain, ${ }^{35}$ but IL-4R $\alpha$ and IL-13R $\alpha$ l form a functional receptor complex that binds both IL-4 and IL$13 .^{36}{ }^{37}$ IL- 13 binds with 100 -fold higher affinity for IL-13R $\alpha 2$ than IL-13R $\alpha 1$, but the IL-13R $\alpha 2$ chain does not appear to signal and may actually function as an in vivo inhibitor of IL13 function. ${ }^{38}$ IL-13R $\alpha 2$ does not bind IL- $4 .{ }^{39}$ Thus, the purpose of our study was to examine the expression of IL-4 and IL-13 receptor subunits in SLBs from patients with IIP and normal controls. Our findings demonstrate that receptor subunits that bind Th2 cytokines, such as IL-4 and IL-13, are greatly increased in SLBs from patients with IIP (particularly UIP) relative to normal patients, thereby supporting earlier studies that found a Th2 cytokine bias in IIP.

\section{METHODS}

\section{Patients}

The institutional review board at the University of Michigan Medical School, USA approved our study and informed consent was obtained from each patient. Table 1 summarises the clinical characteristics of the 39 patients enrolled in our study. Members of the Michigan endstage lung disease network referred these patients upon a suspicion of IIP, as determined from a compilation of symptoms, physiological symptoms, and radiographical findings.

All patients underwent clinical evaluation, including chest radiography, lung function measurements, and high resolution computed tomography (HRCT) before fibreoptic bronchoscopy, as described previously. ${ }^{2}$ Two thoracic radiologists (EAK, BHG) prospectively and independently scored all lobes on HRCT for ground glass opacity (alveolar abnormality) and interstitial opacity (fibrotic abnormality) on a scale of $0-5$, as described previously. ${ }^{40}$ The radiologists were unaware of the histological diagnosis at the time of interpretation.
None of the patients enrolled in our study had undergone previous biopsy surgery or received treatment for IIP. SLBs were performed as part of the evaluation for the University of Michigan Specialized Center of Research in the pathobiology of fibrotic lung disease between May 2000 and August 2001. All biopsy slides were obtained and each lobe assigned a random number and reviewed by two pathologists (TVC, WDT) as described previously. ${ }^{41}$ Initial readings were performed individually without knowledge of other pathologist's interpretations or clinical or radiographical information. Cases without a uniform diagnosis between pathologists were reviewed together to reach a consensus diagnosis. The pathologists assigned a diagnosis of UIP, NSIP, RBILD, or other to each lobe according to established criteria (American Thoracic Society position statement on IIP $^{8}$ ). The profusion of fibroblastic foci (FF) for each lobe was scored semiquantitatively as $0-3$ (absent, 0; mild, 1; moderate, 2; pronounced, 3) by each pathologist, as described recently. ${ }^{42}$ A mean FF score for each patient was calculated by averaging the FF score from each lobe. A maximal FF score was recorded as the highest FF score for any lobe. Histologically normal lung was obtained from resected specimens in five patients undergoing thoracic resection for reasons other than suspicion of IIP. Each biopsy was processed separately using a sterile technique in a laminar flow hood and processed for molecular, protein, and histological analysis. Detailed descriptions of the techniques used in our study are provided below.

\section{Preparation of RNA and cDNA from SLBs}

The portion of each SLB used for molecular analysis was snap frozen in liquid nitrogen and stored at $-80^{\circ} \mathrm{C}$. For RNA isolation, these samples were thawed on ice, and mechanically homogenised in TRIzol ${ }^{\circledR}$ reagent (Invitrogen Life Technologies, Carlsbad, California, USA) and total RNA was then prepared according to the manufacturer's instructions. Purified RNA from SLBs was subsequently reverse transcribed into cDNA using a BRL reverse transcription kit and oligo (dT) 12-18 primers. The amplification buffer contained $50 \mathrm{mM} \mathrm{KCl}, 10 \mathrm{mM}$ Tris/HCl (pH 8.3), and 2.5 $\mathrm{mM} \mathrm{MgCl}_{2}$.

\section{Real time Taqman PCR analysis}

Human IL-4, IL-13, and IFN $\gamma$ gene expression in IIP and normal SLBs was analysed by real time quantitative reverse transcription polymerase chain reaction (RT-PCR) using an ABI PRISM 7700 sequence detection system (Applied Biosystems, Foster City, California, USA). The cDNAs from upper and lower lobe SLBs were analysed for cytokine and

Table 1 Clinical characteristics of the patients studied

\begin{tabular}{|c|c|c|c|}
\hline & UIP $(n=21)$ & NSIP $(n=6)$ & $\mathrm{RB}(\mathrm{n}=8)$ \\
\hline $\begin{array}{l}\text { Sex (M/F) } \\
\text { Age (years) }{ }^{\star} \\
\text { Duration of symptoms (years) } \\
\text { Smoking (\% current or ex-smokers) } \\
\text { FVC (\% of that predicted)† } \\
\text { TLC (\% of that predicted) } \ddagger \\
\text { DLCO (\% of that predicted) } \\
\text { CT-fib } \\
\text { CT-alv } \\
\text { Mean FF§ } \\
\text { Maximum FF- }\end{array}$ & $\begin{array}{l}14 / 7 \\
59(7) \\
2.1(2.4) \\
71 \\
61(19) \\
66(18) \\
44(19) \\
1.57(0.68) \\
1.44(0.89) \\
1.41(0.82) \\
1.59(0.85)\end{array}$ & $\begin{array}{l}4 / 2 \\
61(7) \\
0.6(0.5) \\
50 \\
68(21) \\
77(23) \\
38(17) \\
1.53(1.60) \\
1.13(1.71) \\
0.58(0.51) \\
0.63(0.48)\end{array}$ & $\begin{array}{l}2 / 6 \\
45(11) \\
4.4(4.9) \\
83 \\
72(20) \\
85(21) \\
58(12) \\
0.55(0.07) \\
1.55(1.20) \\
0.17(0.41) \\
0(0)\end{array}$ \\
\hline \multicolumn{4}{|c|}{ 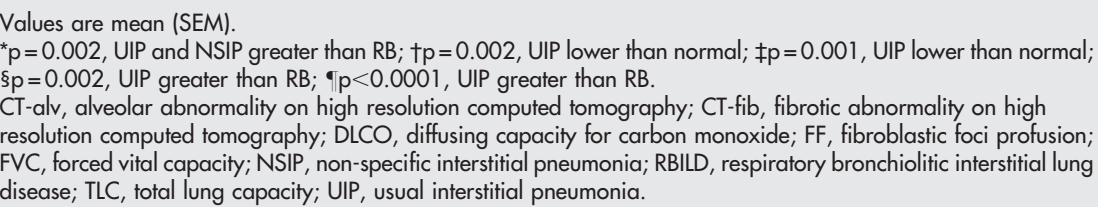 } \\
\hline
\end{tabular}


glyceraldehyde 3-phosphate dehydrogenase (GAPDH; an internal control) expression. All primers and probes used were purchased from Applied Biosystems. Cytokine gene expression was normalised to GAPDH before the "fold" change in cytokine expression was calculated. The fold increases in cytokine gene expression were calculated by comparing gene expression in all patients with that detected in the corresponding upper or lower lobes from the normal patient group. If cytokine gene expression was not detected in a given biopsy within 40 cycles, the sample was assumed to be negative for mRNA encoding this cytokine, and a value of 0 was assigned to the sample for the purposes of calculating fold differences between the IIP patient groups versus the normal patient group.

\section{RT-PCR amplification}

Isolated mRNA from SLBs was also analysed by RT-PCR. Specific oligonucleotide primers were added (200 ng/sample) to the buffer, along with $1 \mu \mathrm{l}$ of reverse transcribed cDNA sample. The following human oligonucleotide primers $\left(5^{\prime} \rightarrow 3^{\prime}\right.$ sequences) were used for RT-PCR analysis: IL-4R $\alpha$ sense, TGCGTCTCCGACTACATGAG; IL-4R $\alpha$ antisense, TGACTGCATAGGTGAGATG (387 bp product); IL-13R $\alpha 1$ sense, AAGGAATACCAGTCCCGACA; IL-13R $\alpha$ l antisense, ACCAGGGAACCATGAAACAAG (457 bp product); IL-13R $\alpha 2$ sense, GTGAAACATGGAAGACCATC; IL-13R $\alpha 2$ antisense, GTGAAATAACTGGATCTGATAGGC (453 bp product). Mixtures containing cDNA and IL-4R $\alpha$, IL-13R $\alpha$ l, or IL$13 \mathrm{R} \alpha 2$ sense and antisense primers were then incubated for four minutes at $94^{\circ} \mathrm{C}$ and amplified using the following cycling parameters: 38 cycles of $94^{\circ} \mathrm{C}$ for 45 seconds, $60^{\circ} \mathrm{C}$ for 60 seconds, and elongation at $72^{\circ} \mathrm{C}$ for 60 seconds. After amplification, the PCR products were separated on a $2 \%$ agarose gel containing $0.3 \mu \mathrm{g} / \mathrm{ml}$ of ethidium bromide and bands were visualised using a molecular imager FX ProPlus (Biorad, Hercules, California, USA). Digitised images of each gel were then analysed using PDQuest analysis software (Biorad) and the ratio of the density of the receptor subunit to $\beta$ actin was then calculated.

\section{ELISA analysis}

Human IL-4, IL-13, and IFN $\gamma$ protein concentrations were measured in $50 \mu \mathrm{l}$ cell free supernatant samples from homogenised IIP and normal SLBs using a standardised sandwich enzyme linked immunosorbent assay (ELISA) technique (R\&D Systems, Minneapolis, Minnesota, USA). Recombinant human cytokines were used to generate the standard curves from which the concentrations present in the samples were derived. The limit of detection for IL-4, IL-13, and IFN $\gamma$ in these ELISAs was consistently above $2 \mathrm{pg} / \mathrm{mg}$ of protein. The cytokine values in each sample were normalised to total protein concentrations in each sample using the Bradford assay.

\section{Immunohistochemistry}

Paraffin wax embedded SLBs were analysed for immunohistochemical localisation of IL-4R $\alpha$, IL-13R $\alpha$ l, and IL-13R $\alpha 2$. All antibodies directed against these receptor subunits were obtained from R\&D Systems. Histological sections $(5 \mu \mathrm{m}$ thick) were dewaxed with xylene, rehydrated in graded concentrations of ethanol, and blocked with normal rabbit serum (Vectorstain ABC-AP kit; Vector Laboratories, Burlingame, California, USA). Goat antihuman IL-4R $\alpha$, IL13R $\alpha 1$, IL-13R $\alpha 2$ antibodies, and control normal goat IgG, were diluted in phosphate buffered saline to a final concentration of $2 \mu \mathrm{g} / \mathrm{ml}$. Antibodies or IgG were added to histological sections for 60 minutes, after which each tissue section was washed thoroughly three times with phosphate buffered saline. A secondary rabbit antigoat biotinylated antibody (Vector Laboratories) was added to each section for 60 minutes, after which each slide was thoroughly washed and either alkaline phosphatase (Vector Laboratories) or peroxidase (R\&D Systems) conjugated to avidin was added to each. Receptor localisation was revealed with an alkaline substrate, 5-bromo, 4-chloro, 3-indolyphosphate/nitroblue tetrazolium (BCIP/NBT), or with 3-amino-9-ethylcarbazole (AEC). Coverslips were applied to each slide using an aqueous mounting solution. A total of 12 patients with UIP (upper and lower lobe histological samples) and four patients from each of the other IIP and normal groups (upper and lower lobe histological samples) were examined for the presence of IL-4 and IL-13 receptor subunit expression.

\section{Statistical analysis}

All results are expressed as mean (SEM). ANOVA analysis and the Kruskal-Wallis or the Tukey-Kramer multiple comparison tests were used to reveal significant differences among and between the IIP and normal patient groups. The data were presumed to be non-parametric because of the inclusion of gene (by Taqman) and protein (by ELISA) values that were below the limit of detection, and thus assigned a value of zero. $p$ Values $<0.05$ were considered significant.

\section{RESULTS}

Pathological and clinical features of patients studied Thirty nine patients were enrolled in our study and table 1 summarises the baseline pathological and clinical characteristics of the three IIP groups examined. Twenty one of the patients exhibited histological features consistent with UIP; 20 of these patients had concordant UIP whereas one had NSIP in one lobe and UIP in another lobe. ${ }^{41}$ The NSIP cases included six patients further subclassified as two patients with a cellular pattern and four with a fibrotic variant. As expected, the patients with UIP exhibited a lower FVC and TLC than did those with NSIP or RBILD. In addition, patients with UIP had greater numbers of fibroblastic foci. Upper and/ or lower SLBs from normal patients $(n=5)$ were used as controls, and none of these SLBs showed histological evidence of pulmonary disease. The sex distribution of this normal group was four men and one woman, with the average age being 53 years (age range, 21-83).

\section{IL-4, IL-13, and IFN $\gamma$ gene expression in IIP and normal SLBs}

Figure 1 shows the results of quantitative Taqman PCR analysis of the IL-13, IL-4, and IFN $\gamma$ genes in SLBs from all three patient groups. IL-13 gene expression was increased approximately 27 -fold and 31 -fold in upper and lower SLBs, respectively, in patients with UIP compared with the normal patient group (fig lA) ( $p<0.0001$ ). IL-13 gene expression was also increased approximately 17 -fold and twofold in upper and lower SLBs, respectively, in patients with NSIP. In the RBILD patient group, IL-13 gene expression was increased approximately sevenfold in upper SLBs, although there was no increase in lower SLBs from this patient group. There were no significant differences in IL-13 gene expression among the IIP groups analysed.

Quantitative IL-4 gene expression differed substantially in all IIP patient groups from that seen for IL-13 (fig 1B). Once again, the changes in IL-4 gene expression were relative to the reference normal patient group. Both upper and lower SLBs from the patients with UIP showed a significantly smaller increase in IL-4 gene expression than did the corresponding SLBs in the NSIP group. A significantly greater increase in IL-4 gene expression was also seen in the upper SLBs of the NSIP group than in the patients with RBILD. IL-4 gene expression in the upper and lower SLBs from patients 

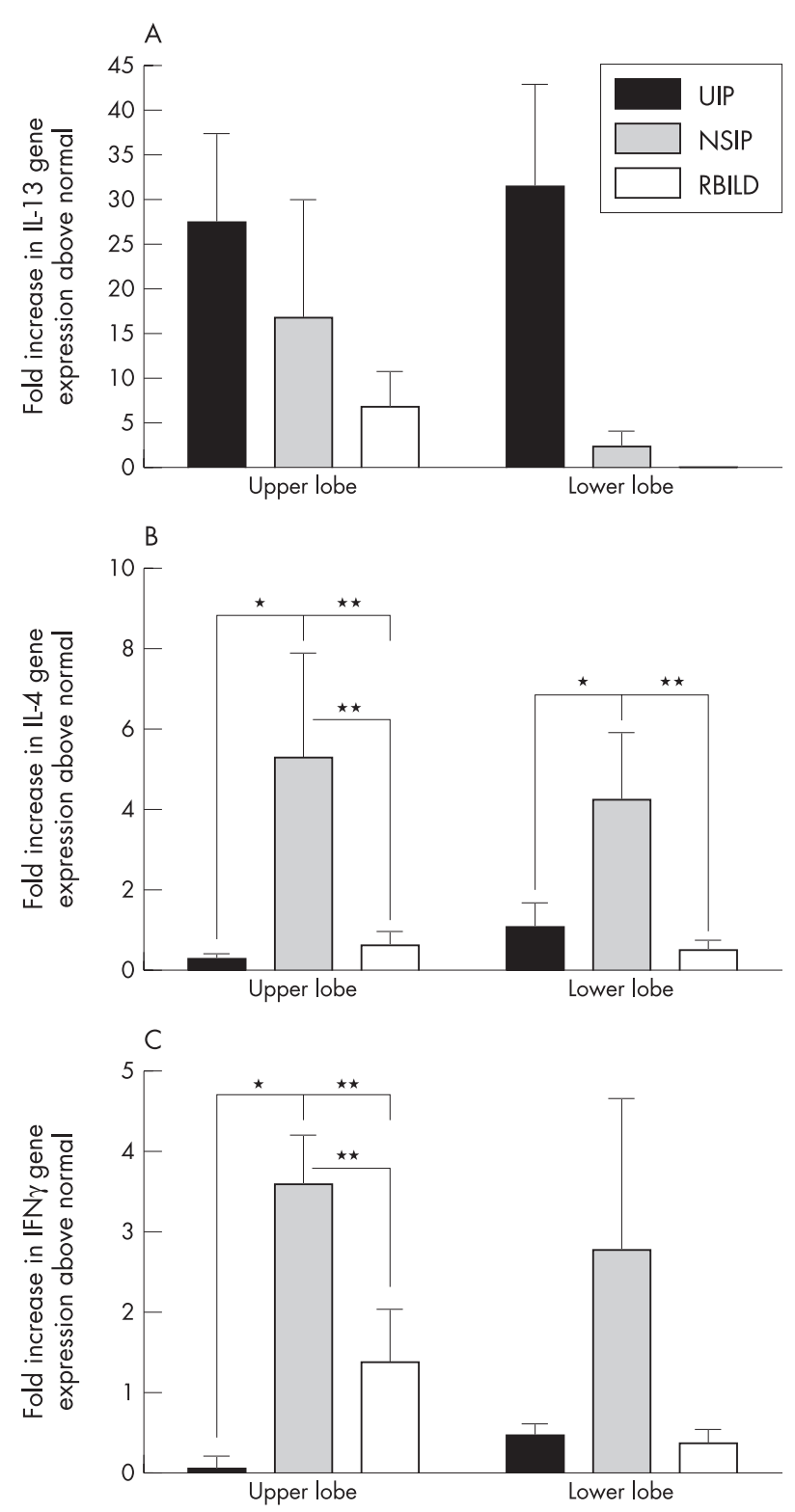

Figure 1 Quantitative Taqman polymerase chain reaction analysis of upper and lower surgical lung biopsies (SLBs) from patients with usual interstitial pneumonia (UIP), non-specific interstitial pneumonia (NSIP), and respiratory bronchiolitic interstitial lung disease (RBILD). Data shown are means of the fold increase in (A) interleukin 13 (IL-13), (B) IL-4, and (C) interferon $\gamma$ (IFN $\gamma$ ) gene expression in each idiopathic interstitial pneumonia (IIP) patient group above that detected in the appropriate lobe from the normal patient group. ${ }^{*} p \leqslant 0.05,{ }^{* *} p \leqslant 0.001$ compared with the indicated upper or lower lobe SLBs from other IIP patient groups.

with NSIP was approximately fivefold and fourfold higher, respectively, than that detected in the control patients.

Increases in IFN $\gamma$ gene expression in upper SLBs were significantly greater in patients with NSIP than in those with UIP (fig $1 C$ ). The changes in IFN $\gamma$ gene expression were relative to the normal patient group. Among upper and lower SLBs, the greatest increases in IFN $\gamma$ gene levels were seen in patients with NSIP (upper, 3.5-fold increase; lower, 2.7-fold increase). In the RBILD patient group, IFN $\gamma$ gene expression was increased approximately 1.4-fold in upper lobe biopsies. Significant differences were detected in the upper lobe SLBs from patients with NSIP compared with those from patients with UIP and RBILD (fig $1 \mathrm{C}$ ). Thus, quantitative Taqman analysis showed that the SLBs from patients with UIP were skewed in their cytokine transcript expression towards IL-13 and away from IL-4 and IFN- $\gamma$, whereas SLBs from patients with NSIP showed a balanced Thl/Th2 cytokine transcript profile.

\section{IL-4, IL-13, and IFN $\gamma$ protein expression in IIP and normal SLBs}

Figure 2 shows the concentrations of IL-13, IL-4, and IFN $\gamma$ proteins detected by ELISA in upper and lower SLBs from the IIP and normal groups. Similar concentrations of IL-13 were seen in upper SLBs from all IIP groups examined, but these values were approximately 50-150 times higher in the IIP groups than in the normal control group (normal values are indicated by the solid line in fig 2). IL-13 concentrations in both upper and lower SLBs from the RBILD group were significantly $(p \leqslant 0.05)$ higher than those detected in the normal SLBs. In addition, significantly $(p \leqslant 0.01)$ more IL13 was detected in the lower SLBs from patients with RBILD compared with those with UIP (fig 2A). IL-4 concentrations were significantly higher in upper and lower SLBs from patients with UIP than in the corresponding lobes from patients with RBILD and controls (fig 2B). The pattern of expression of the IFN $\gamma$ protein mirrored that of the IFN $\gamma$ gene in the IIP patient groups (fig 2C). Although no significant differences were seen among the patient groups, the lowest IFN $\gamma$ protein concentrations were detected in the upper and lower SLBs from the UIP patient group. IFN $\gamma$ protein concentrations were below the limits of detection by ELISA in the upper and lower SLBs from the normal patient group. Thus, these data suggested that IL-4, IL-13, and IFN $\gamma$ protein concentrations in IIP SLBs differed greatly from those seen in normal SLBs. In addition, IL-13 was the predominant cytokine in SLBs from patients with RBILD, whereas IL-4 predominated in SLBs from patients with UIP and NSIP.

\section{IL-4R $\alpha$, IL- 13R $\alpha$ 1, and IL-13R 2 gene expression in IIP and normal SLBs}

Figure 3 summarises the results of the densitometry analyses of RT-PCR products corresponding to IL-13R $\alpha 2$, IL-13R $\alpha 1$, and IL-4R $\alpha$ and $\beta$ actin in IIP upper and lower SLBs. The data shown are the ratio of the density of the receptor subunit PCR product to that of the corresponding $\beta$ actin PCR product in the same sample. It was apparent from this semiquantitative analysis that the expression of all three receptor subunit transcripts was higher in upper and lower SLBs from the patients with UIP than the normal patients, although this increase did not reach significance. In contrast, upper lobe IL$13 R \alpha 2$ transcript values were significantly lower in the NSIP and RBILD patient groups than in normal biopsies. Furthermore, IL-13R $\alpha$ l transcript values were significantly lower in upper and lower lobe SLBs from the patients with NSIP compared with the normal group. The mean and SEM values for the normal group are shown by the solid and dashed lines, respectively, in fig 3. Thus, the expression of IL4 and IL-13 receptor subunit transcripts varied among the IIP and normal patient groups examined in our study.

\section{IL-4R $\alpha$, IL- $13 R \alpha 1$, and IL- $13 R \alpha 2$ protein expression in IIP and normal SLBs}

The RT-PCR analysis summarised above suggested that the expression of IL-4 and IL-13 receptor subunit transcripts was enhanced in the UIP patient group. To examine whether increased receptor transcript values corresponded with increased receptor protein expression, we used routine immunohistochemical staining to examine histological sections from formalin fixed SLBs. Our immunohistochemical analysis indicated that both IL-13 receptor subunits were expressed abundantly in SLBs from the IIP patient groups, 

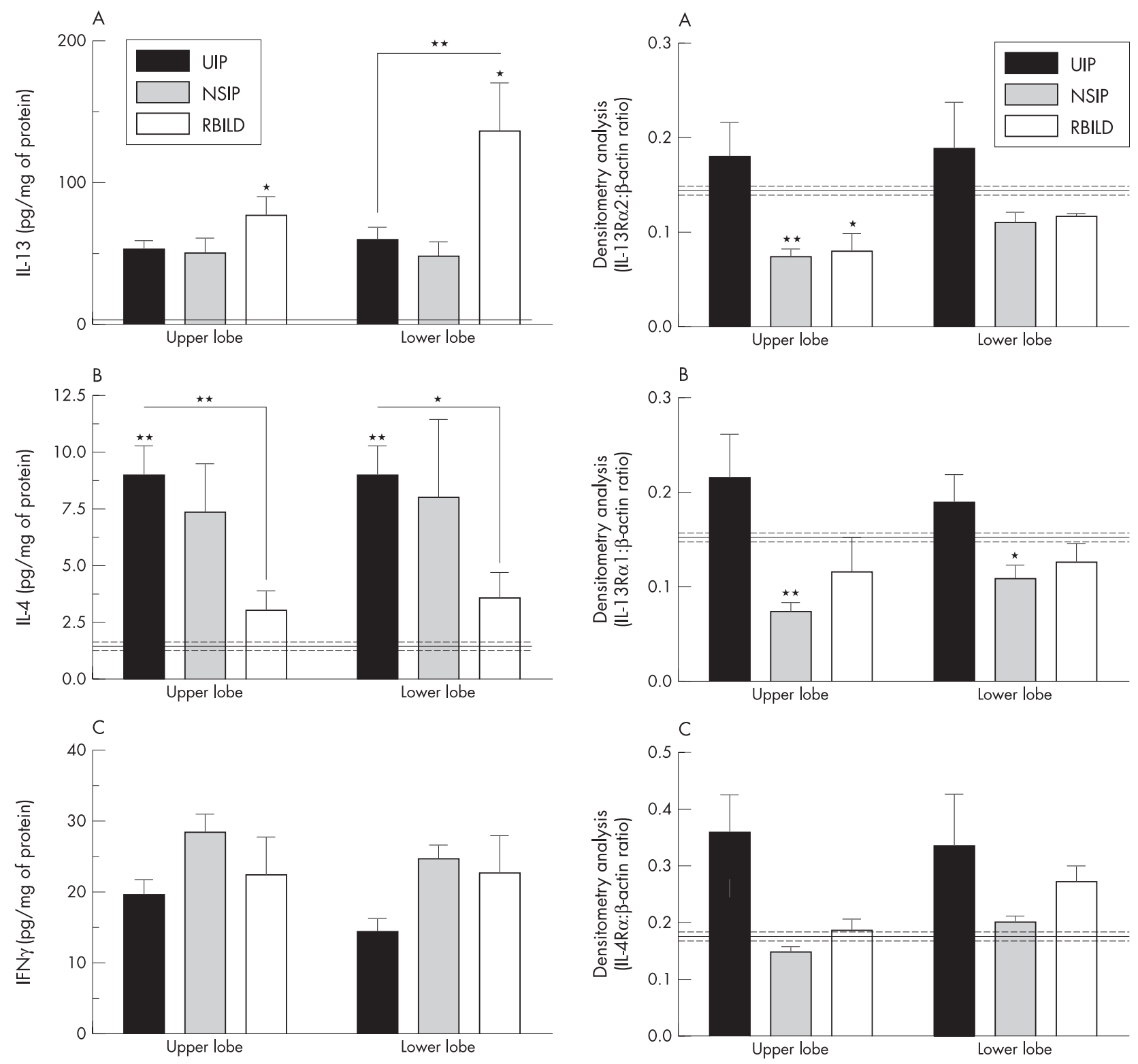

Figure 2 Enzyme linked immunosorbent assay analysis of $(A)$ interleukin 13 (IL-13), (B) IL-4, and (C) interferon $\gamma$ (IFN $\gamma$ ) protein concentrations in surgical lung biopsies (SLBs) from patients with idiopathic interstitial pneumonia (IIP) (bars) and normal patient groups (solid (mean level) and dashed lines (SEM)). Data are mean \pm SEM; ${ }^{*} p \leqslant 0.05,{ }^{* *} p \leqslant 0.01$ compared with the indicated upper or lower lobe SLBs from all other IIP and normal patient groups (asterisks above the bars). Note that cytokine values were identical in upper and lower lobe samples from the normal patient group so that a single mean and SEM is shown for this group. NSIP, non-specific interstitial pneumonia; RBILD, respiratory bronchiolitic interstitial lung disease; UIP, usual interstitial pneumonia.

and representative staining for IL-13R $\alpha$ l and IL-13R $\alpha 2$ is shown in figs 4 and 5, respectively. We found that both IL-13 receptor subunits stained strongly in upper lobe SLB histological sections from the patients with RBILD (figs 4A, $\mathrm{B}$ and $5 \mathrm{~A}, \mathrm{~B})$. No staining for either subunit was seen in lower lobe SLBs from patients with RBILD (not shown). IL$13 \mathrm{R} \alpha \mathrm{l}$ expression was associated with blood vessels in histological sections from the patients with NSIP (fig 4C, D), whereas interstitial areas of the lung were lightly stained for the IL-13R $\alpha 2$ subunit (fig 5C, D). In upper and lower lobe SLBs from the UIP patient group, staining for IL-13R $\alpha$ l was

Figure 3 Densitometry analysis of reverse transcription polymerase chain reaction products of the (A) interleukin 13 receptor $\alpha 2$ (IL-13R $\alpha 2$ ), (B) IL-13R $\alpha 1$, and (C) IL-4R $\alpha$ genes in upper and lower surgical lung biopsies (SLBs) from patients with idiopathic interstitial pneumonia and controls (mean control values, solid line; control SEM values, dashed lines). Data are means \pm SEM; ${ }^{*} p \leqslant 0.05,{ }^{* *} p \leqslant 0.01$ compared with the control SLBs. Note that cytokine receptor transcript values were identical in upper and lower lobe samples from the normal patient group so that a single mean and SEM value is shown for the normal patient group. NSIP, non-specific interstitial pneumonia; RBILD, respiratory. bronchiolitic interstitial lung disease; UIP, usual interstitial pneumonia.

particularly strong on many blood vessels present in interstitial areas of the lung (fig 4E, F), whereas very strong staining for IL-13R $\alpha 2$ was seen in the lung epithelium, interstitium of the lung (fig 5E, F), and in mononuclear cells (inset to fig 5E).

\section{Foci or localised expression of IL-4R $\alpha$ and IL- 13R 2 in UIP and NSIP SLBs}

We next examined whether IL-4 and IL-13 receptor subunits were associated with distinct fibroblastic foci in SLBs from patients with IIP, particularly patients with UIP. No focal patterns of IL-13R $\alpha$ l subunit expression were seen in the IIP 


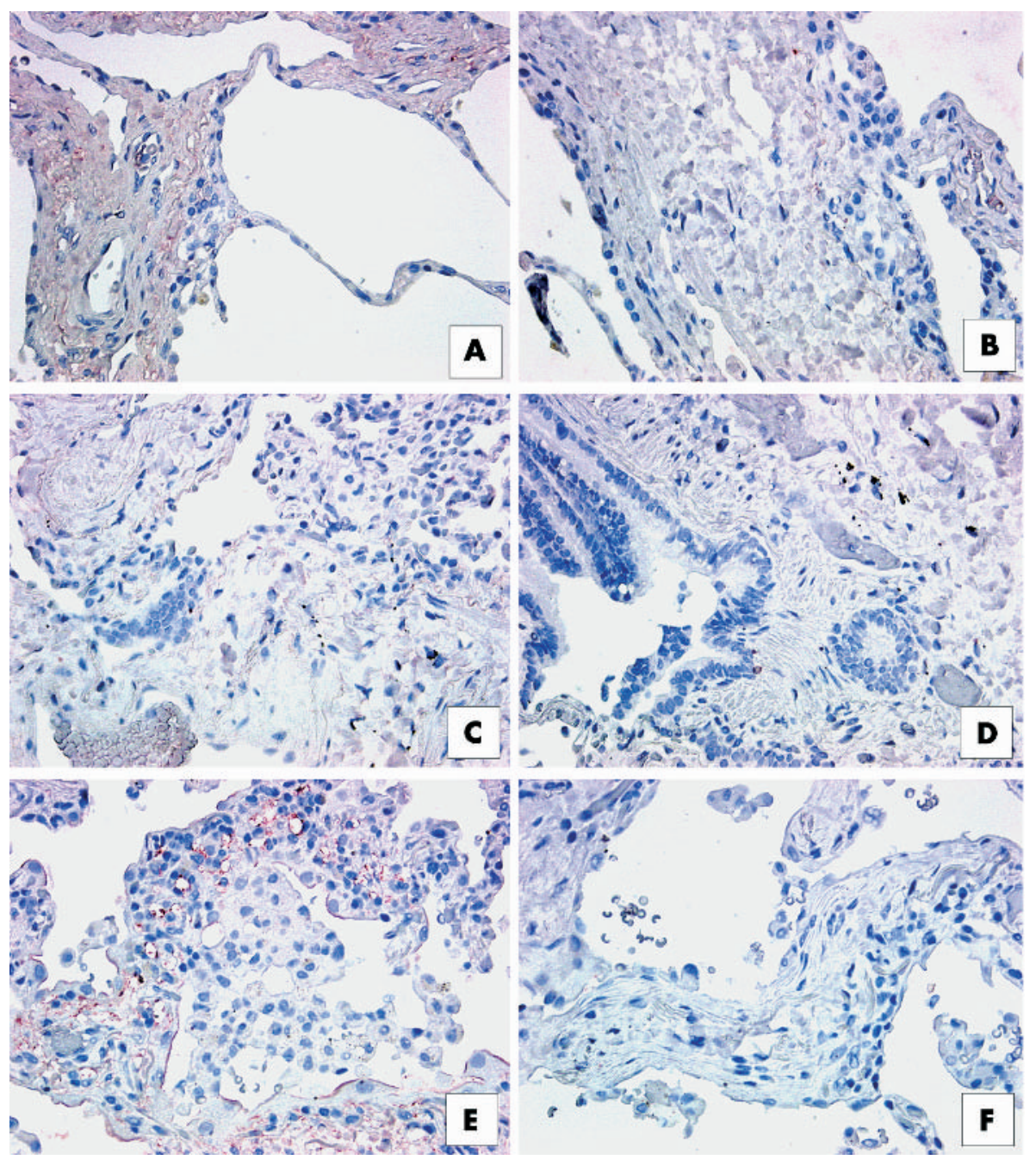

Figure 4 Immunohistochemical analysis of interleukin 13 receptor $\alpha 1$ (IL-13R $\alpha$ ]) in upper lobe surgical lung biopsies (SLBs) from patients with (A, B) respiratory bronchiolitic interstitial lung disease, $(C, D)$ non-specific interstitial pneumonia (NSIP), and (E, F) usual interstitial pneumonia (UIP).

Representative positive (red) staining for IL-13R $\alpha 1$ is shown in panels (A), (C), and (E). Appropriate negative controls are shown in panels (B), (D), and (F). (A) IL-13R $\alpha 1$ was expressed abundantly in the interstitium of SLBs from patients with RBILD. (B) IL-13R $\alpha$ l expression was associated with blood vessel walls in histological sections from patients with NSIP, whereas interstitial areas were lightly stained for this subunit. (E) IL$13 R \propto 1$ (red staining) was particularly prominent in pulmonary areas of neovascularisation in SLBs from patients with UIP.

biopsies analysed immunohistochemically. As shown in fig 6, IL-4R $\alpha$ protein expression was prominent in distinct foci present in upper lobe SLBs from a patient with UIP (fig 6B) and a patient with NSIP (fig 6E). Based on an examination of haematoxylin and eosin stained sections, we suspect that these foci of intense IL- $4 \mathrm{R} \alpha$ staining may correspond to fibroblastic foci. Expression of the IL- $4 \mathrm{R} \alpha$ subunit protein was seen only in immune cells (mononuclear cells and alveolar macrophages) present in the histological sections from a patient with RBILD (fig $6 \mathrm{H}$ ) and a normal patient (fig $6 \mathrm{~K}$ ). Considerably fewer IL-4R $\alpha$ positive immune cells were seen in the normal SLBs compared with the RBILD SLBs.

Large foci of cells positive for IL-13R $\alpha 2$ protein were seen in equal abundance in both upper and lower lobes from the patients with UIP (fig 6C). However, foci of cells positive for IL-13R $\alpha 2$ were not seen in either the upper or lower lobe biopsy samples from the patients with NSIP. Instead, we found a lightly diffuse pattern of IL-13R $\alpha 2$ staining (fig 6F), similar to that shown in fig 5C. In the RBILD patient group, we found that IL-13R $\alpha 2$ was expressed in immune cells (mononuclear cells or alveolar macrophages) and in some interstitial areas of upper lobe SLBs (fig 6I), but no foci were seen. Scattered mononuclear and alveolar macrophages stained for the IL-13R $\alpha 2$ protein (fig $6 \mathrm{~L}$ ), but this receptor subunit was rarely found in other areas of normal SLBs. Thus, these immunohistochemical data demonstrate that severe forms of IIP, such as UIP and NSIP, are associated with increased IL-4R $\alpha$ and IL-13R $\alpha 2$ protein expression within distinct foci, which appear to be primarily composed of fibroblasts, particularly in UIP SLBs.

\section{DISCUSSION}

Surgical lung biopsies are important in the diagnosis of patients suspected of having IIP. ${ }^{43}$ Therefore, biopsies and other samples from these patients have been studied extensively for clues to the aetiopathogenesis of IIP. We found that IL-4 and IL-13 receptor subunits were expressed at the protein level in SLBs from patients with UIP and NSIP. Specifically, a major finding was the localisation of IL-4R $\alpha$ and IL-13R $\alpha 2$ receptor subunits to distinct foci in upper and lower biopsies from patients with UIP, and the focal expression of IL- $4 \mathrm{R} \alpha$ receptor subunits in SLBs from patients with NSIP. Detailed histological examination of these SLBs in patients with UIP revealed that the foci were composed of fibroblasts, and many could be classified as fibroblastic foci. Together, these data suggest that various pathologically defined forms of IIP are associated with a unique focal pattern of expression of IL-4 and IL- 13 receptor subunits, and this observation may be of diagnostic importance in this group of diseases.

"Quantitative Taqman analysis showed clearly that cytokine transcripts differed greatly between the idiopathic interstitial pneumonia patient groups analysed" 


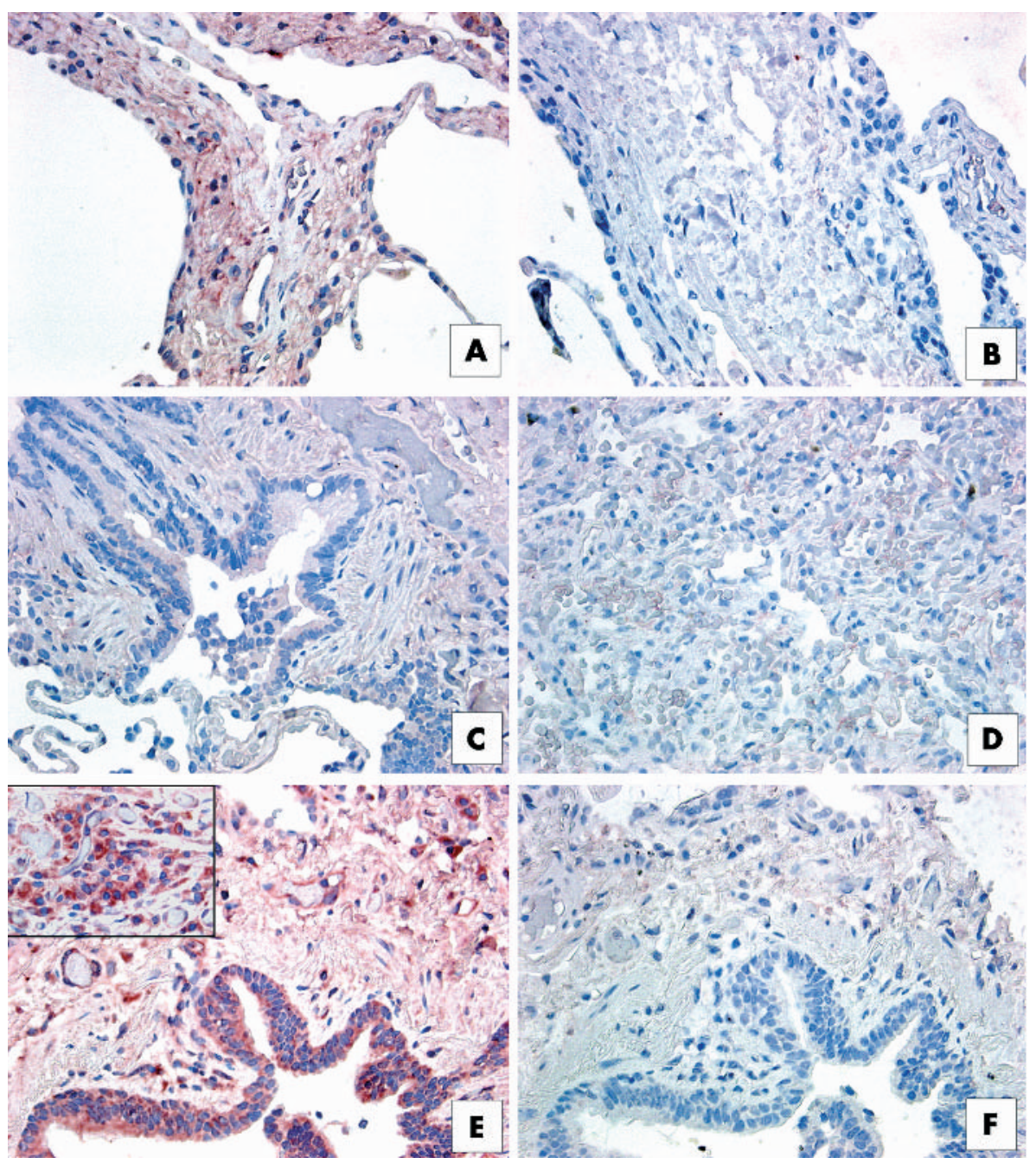

Figure 5 Immunohistochemical analysis of interleukin 13 receptor $\alpha 2$ (IL-13R $\alpha 2$ ) in upper lobe surgical lung biopsies (SLBs) from patients with (A, B) respiratory bronchiolitic interstitial lung disease, $(C, D)$ non-specific interstitial pneumonia (NSIP), and (E, F) usual interstitial pneumonia (UIP). Representative positive (red) staining for IL-13R $\alpha 2$ is shown in panels (A), (C), and (E). Appropriate negative controls are shown in panels (B), (D), and (F). (A) Strong interstitial (red) staining for IL-13R $\alpha 2$ was seen in SLBs from patients with RBILD. (C) IL-13R $\alpha 2$ was lightly stained in interstitial areas in SLBs from patients with NSIP. (E) In contrast, very intense IL-13R $\alpha 2$ expression (red staining) was detected in the lung epithelium, interstitium, and in mononuclear cells present within SLBs from patients with UIP.

Several previous studies of biopsy material from patients with UIP/IPF have shown that the overall cytokine pattern is biased towards a Th2 rather than a Thl response, ${ }^{14}{ }^{16}{ }^{17}$ in contrast to other lung diseases, such as sarcoidosis, which exhibits a strong Thl profile. ${ }^{44}$ Also consistent with the Th2 cytokine bias in IPF are the findings of Smith et al, ${ }^{45}$ who reported that lung IL-1 receptor antagonist protein (IRAP) content was increased in tissue homogenates and bronchoalveolar lavage fluid from patients with IPF compared with normal individuals. Both IL-4 and IL-13 are potent activators of IRAP expression in macrophages ${ }^{46}$ It was clear from our Taqman analysis that the expression of IL-4, IL-13, and IFN $\gamma$ transcripts differed significantly among IIP patient groups, and these data closely agreed with previous studies showing a bias towards the expression of genes encoding Th2 cytokines (IL-4 and IL-13) rather than Thl (IFN- $\gamma$ ) cytokines in IPF/UIP. ${ }^{14}{ }^{16}{ }^{17}$ The expression of IL-13 transcripts has not been examined previously in SLBs from IIP patient groups, and our study showed that the expression of this Th2 cytokine is greatly increased in UIP compared with SLBs in the other IIP groups and normal controls. In addition, this Th2 cytokine was selectively increased in UIP SLBs because IL-4 expression was not increased to the same degree. This was in contrast to NSIP SLBs, where the expression of IL-4 transcripts was increased significantly. The major Thl cytokine, IFN $\gamma$, was higher in upper and lower NSIP biopsies compared with upper and lower SLBs from the other IIP groups, and this increase was significant in upper lobe biopsies. Together, quantitative Taqman analysis showed clearly that cytokine transcripts differed greatly between the IIP patient groups analysed.

Using specific ELISA analysis of homogenised upper and lower lobe biopsies, we investigated whether Thl and Th2 cytokines differed at the protein level in IIP biopsies relative to normal biopsies. Significant differences in IL-13 and IL-4, but not IFN $\gamma$, protein concentrations were found in IIP SLBs compared with normal biopsies. However, in contrast to the Taqman analysis, IL-13 was found in greatest abundance in RBILD biopsies compared with UIP and NSIP biopsies. The reason for this discrepancy between transcript and protein expression is unclear, but it may reflect the differences in IL13R $\alpha 2$ expression seen among these IIP biopsies. Specifically, our ability to detect differences in IL-13 values in homogenates of IIP and normal SLBs by ELISA may have been hampered by the presence of soluble isoforms of the IL-13 receptor subunits. Further differences between the amounts of IL- 4 and IFN $\gamma$ transcripts and proteins were also seen in upper and lower lobe biopsies from patients with UIP. Once again, an explanation for this disparity between gene and protein values is not apparent, but it may result from a defect in cytokine regulation in this severe form of IIP. Gene and protein values relative to normal corresponded more closely in NSIP and RBILD biopsies. Overall, our protein findings confirm that IL-13 and IL-4 were greatly increased in IIP SLBs compared with normal SLBs, but we also found that IFN $\gamma$ was abundant in IIP biopsies. This last finding is in contrast to other studies, but it might be explained by the fact that previous studies looking at cytokine patterns in UIP 


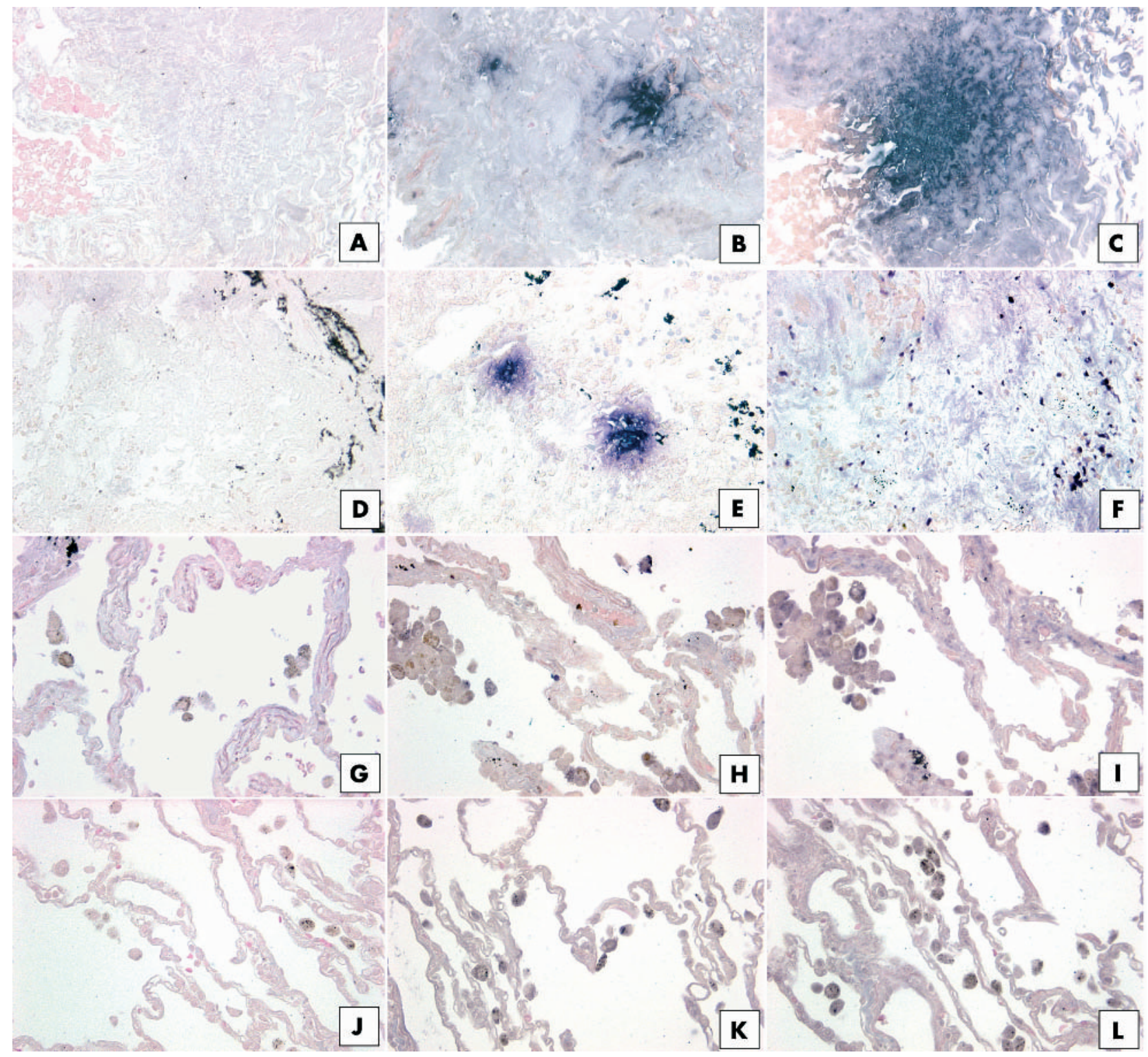

Figure 6 Immunohistochemical analysis of $(B, E, H, K)$ interleukin 4 receptor $\alpha(I L-4 R \alpha)$ and $(C, F, I, L) ~ I L-13 R \alpha 2$ in upper lobe surgical lung biopsies (SLBs) from patients with (B, C) usual interstitial pneumonia (UIP), (E, F) non-specific interstitial pneumonia (NSIP), (H, I) respiratory bronchiolitic interstitial lung disease (RBILD) and (K, L) normal controls. Representative positive (purple) staining for IL- $4 R \alpha$ and IL-13R $\alpha 2$ was detected in distinct foci in UIP SLBs (B, C), and IL-4R $\alpha$ was also detected in distinct foci in NSIP SLBs (E). (H) Only mononuclear cells expressed IL-4R $\alpha$ protein in upper SLBs from patients with RBILD. (I) Staining of mononuclear and interstitial cells for IL-13 R $\alpha 2$ was seen in upper SLBs from patients with RBILD. (C) IL-13R $\alpha 2$ was lightly stained in interstitial areas in SLBs from patients with NSIP. Rare IL-4R $\alpha(K)$ and IL-13R $\alpha 2$ (L) positive mononuclear cells were seen in SLBs from the normal controls. (A, D, G, J) Negative controls for each patient group. Black material (D, E, F) may be deposits as a result of cigarette smoke.

SLBs used immunohistochemical approaches to identify interstitial areas that were positive either for IL-4 or IFN $\gamma .{ }^{14}{ }^{16}$ Other previous studies showing a Th2 bias in patients with IPF/UIP reached this conclusion using isolated monocytes, lymphocytes, or alveolar macrophages. ${ }^{153347}$ Although the difference did not reach significance, we found that IFN $\gamma$ protein values were lower in UIP than in NSIP and RBILD upper and lower SLBs. It should also be noted that there are several concerns about the measurement of cytokine proteins in whole lung homogenates, such as the presence of soluble decoy receptors and post-translational modifications to cytokine proteins, which render them undetectable by the ELISAs used in our study.

UIP is the most common histological pattern seen in cases of IIP. ${ }^{48}$ The disease is characterised by a rapid progression to death, with an average survival of approximately three years after diagnosis. An important histological feature of UIP is the localised areas of fibroblastic proliferation referred to as fibroblastic foci. ${ }^{42}{ }^{49}$ These foci are thought to be sites where repeated and chronic lung damage has occurred. The major finding of our study was that fibroblastic foci stained intensely for IL-4R $\alpha$ and IL-13R $\alpha 2$. Although it has been shown that fibroblastic foci tend to be more abundant in the lower lobes of UIP biopsies, we found that focal staining for the IL-13R $\alpha 2$ and IL-4R $\alpha$ subunits was equally abundant in both the upper and lower lobes, suggesting that staining for these receptor subunits may assist in the identification of these unique histological features. However, this finding may reflect the fact that our UIP group almost entirely comprised patients with concordant disease. At present, it is not clear why the greatly increased expression of the IL-13R $\alpha 2$ receptor subunit gene and protein, particularly in UIP SLBs, fails to 
curtail the profibrotic actions of IL-13. However, it is possible that the natural antagonism of IL-13R $\alpha 2$ is lost in patients with IIP as a result of post-translational modifications of this decoy receptor. It is also possible that, as a result of these modifications, the IL-13R $\alpha 2$ subunit takes on an abnormal cell signalling role in UIP. Ongoing studies with fibroblasts grown from IIP and normal SLBs are now directed at characterising the expression of and the regulatory roles of IL- 4 and IL-13 receptor subunits on these cells. The increase in IL-13R $\alpha 2$ receptor subunit expression by primary pulmonary fibroblasts from IIP SLBs appears to make these cells remain responsive to the exogenous presence of IL-4 and IL-13 compared with normal primary fibroblast lines.

\begin{abstract}
"The most surprising finding in our study was the pronounced expression of IL-13 protein, and the IL-4 and IL-13 receptor subunits in surgical lung biopsies from patients with respiratory bronchiolitic interstitial lung disease, which is a lung disease found predominately in heavy smokers"
\end{abstract}

NSIPs form a group of interstitial pneumonias that are generally associated with a better prognosis than UIP. ${ }^{811-13}$ In our study, we noted distinct differences between SLBs from the NSIP and UIP patient groups. Molecular analysis of upper and lower SLBs showed that IL-4 and IFN $\gamma$ gene expression was significantly higher in patients with NSIP compared with those with UIP, yet IL-13 gene expression was lower. Although higher IFN $\gamma$ protein values were detected in NSIP SLBs compared with UIP SLBs, this increase was not significant. Expression of the IL-4 and IL-13 receptor genes was lower in the NSIP group than in normal controls. The immunohistochemical analysis of NSIP SLBs confirmed, in part, these molecular findings-less IL-4 and IL-13 receptor subunit staining was seen in NSIP biopsies than in UIP biopsies. However, it was interesting to note similar focal expression of the IL- $4 \mathrm{R} \alpha$ protein in biopsies from the NSIP and UIP patient groups. This focal expression of the IL-4 receptor subunit has never been seen in patients with RBILD or normal controls. Thus, our study revealed clear differences and similarities between UIP and NSIP, which support the notion that NSIP represents a separate clinical IIP entity.

The most surprising finding in our study was the pronounced expression of IL-13 protein, and the IL-4 and IL-13 receptor subunits in SLBs from the patients with RBILD, which is a lung disease found predominately in heavy smokers. The alveolar walls of patients with RBILD are often

\section{Take home messages}

- Interleukin 13 receptor $\alpha 2$ (IL-13R $\alpha 2$ ), IL-13R $\alpha 1$, and $\mathrm{IL}-4 \mathrm{R} \alpha$ transcripts were present in a greater proportion of upper and lower lobe biopsies from patients with usual interstitial pneumonia than those with nonspecific interstitial pneumonia and respiratory bronchiolitic interstitial lung disease or normal controls

- Although the implications of this increased IL-4 and IL13 receptor subunit expression remain to be explored, increased receptor expression may facilitate increased tissue responsiveness to the presence of both cytokines

- These findings may be of particular importance in understanding the pathogenesis of idiopathic interstitial pneumonia and, more importantly, may provide important novel therapeutic targets mildly thickened by fibrous tissue, and the degree of interstitial inflammation is often mild to moderate in SLBs from these patients. ${ }^{80}$ The accumulation of alveolar macrophages is often another feature of this disease. Among all patient groups, both upper and lower SLBs from the patients with RBILD showed the highest expression of the IL-13 protein, and this increase was significant for both sites compared with the respective lobe from the normal controls. The cellular source of IL-13 in RBILD is not presently known, although alveolar macrophages are an important source of this cytokine in IIP. ${ }^{33}$ Molecular and immunohistochemical analysis confirmed that IL-4R $\alpha$, IL-13R $\alpha 1$, and IL-13R $\alpha 2$ were present in SLBs from this group, but we noted a clear discrepancy between IL-13 receptor subunit expression in upper versus lower lobes. In fact, the IL-13R $\alpha 1$ and IL-13R $\alpha 2$ proteins were absent from the lower lobes, but were expressed abundantly in upper lobes in this patient group. The lack of IL-13R $\alpha 2$ expression in the lower lobes may have accounted for the grossly increased IL-13 values in the SLBs from this patient group.

In conclusion, we found a pronounced increase in the expression of receptors that respond to IL- 4 and IL- 13 in SLBs from patients with UIP relative to patients with other forms of IIP and normal controls. The implications of this increased IL-4 and IL-13 receptor expression in IIP remain to be explored, but it is possible that increased receptor expression facilitates increased tissue responsiveness to the presence of both cytokines. In light of recent clinical observations that the administration of IFN $\gamma$ to patients with IIP results in substantial improvement, ${ }^{51}$ it is conceivable that new drugs directed towards receptors that bind IL-4 and IL-13 may also have therapeutic effects in these patients. This therapeutic approach has already shown promise in experimental models of fibrosis through the use of a soluble version of IL-13R $\alpha 2^{52}$ or an IL-13-immunotoxin chimaeric protein, which specifically targets and kills IL- 4 and IL- 13 responsive cells in the lung. ${ }^{27} 53$ Additional studies are currently under way to determine whether fibroblasts cultured from SLBs also exhibit differential IL-4 and IL-13 receptor expression, and to explore the synthetic and proliferative consequences of this expression.

\section{Authors' affiliations}

C Jakubzick, E S Choi, S L Kunkel, H Evanoff, C M Hogaboam, Department of Pathology, University of Michigan Medical School, Ann Arbor, Ml 48109-0602, USA

F J Martinez, K R Flaherty, G B Toews, Division of Pulmonary and Critical Care Medicine, Department of Medicine, University of Michigan Medical School

E A Kazerooni, B H Gross, Department of Radiology, University of Michigan Medical School

R K Puri, Laboratory of Molecular Tumor Biology, Division of Cellular and Gene Therapies, Center for Biologics Evaluation and Research, Food and Drug Administration, Bethesda, MD 20852-1448, USA T V Colby, Mayo Clinic, Scottsdale, AZ 85259, USA

W D Travis, Armed Forces Institute of Pathology, Washington, DC 20306-6000, USA

\section{REFERENCES}

1 Douglas W, Ryu J, Schroeder D. Idiopathic pulmonary fibrosis. Impact of oxygen and colchicine, prednisone, or no therapy on survival. Am J Respir Crit Care Med 2000;161:1172-8.

2 Flaherty KR, Toews GB, Lynch JP, et al. Steroids in idiopathic pulmonary fibrosis: a prospective assessment of adverse reactions, response to therapy, and survival. Am J Med 2001;110:278-82.

3 Ryu JH, Colby TV, Hartman TE. Idiopathic pulmonary fibrosis: current concepts. Mayo Clin Proc 1998;73:1085-101.

4 Lasky JA, Brody AR. Interstitial fibrosis and growth factors. Environ Health Perspect 2000;108(suppl 4):751-62.

5 du Bois RM, Wells AU. Cryptogenic fibrosing alveolitis/idiopathic pulmonary fibrosis. Eur Respir J Suppl 2001;32:43s-55s. 
6 Turner-Warwick M. In search of a cause of cryptogenic fibrosing alveolitis (CFA): one initiating factor or many? Thorax 1998;53(suppl 2):S3-9.

7 Travis W, Matsui K, Moss J, et al. Idiopathic nonspecific interstitial pneumonia: prognostic significance of cellular and fibrosing patterns. Am J Surg Pathol 2000;24:19-33.

8 American Thoracic Society, European Respiratory Society (ATS/ERS) international multidisciplinary consensus classification of idiopathic interstitial pneumonias. Am J Respir Crit Care Med 2002;165:277-304

9 Katzenstein A, Myers J. Idiopathic pulmonary fibrosis. Clinical relevance of pathologic classification. Am J Respir Crit Care Med 1998;157:1301-15.

10 Myers JL. NSIP, UIP, and the ABCs of idiopathic interstitial pneumonias. Eur Respir J 1998; 12:1003-4.

11 Daniil Z, Gilchrist F, Nicholson A, et al. A histologic pattern of nonspecific interstitial pneumonia is associated with a better prognosis than usual interstitial pneumonia in patients with cryptogenic fibrosing alveolitis. Am J Respir Crit Care Med 1999;160:899-905.

12 Hampton J, Martinez F, Orens J, et al. Corticosteroids in idiopathic pulmonary fibrosis (IPF): toxicity may outweigh benefits [abstract]. Am J Respir Crit Care Med 1994;149:A878.

13 Flaherty KR, Toews GB, Travis WD, et al. Clinical significance of histological classification of idiopathic interstitial pneumonia. Eur Respir $J$ 2002;19:275-83.

14 Wallace WA, Ramage EA, Lamb D, et al. A type 2 (Th2-like) pattern of immune response predominates in the pulmonary interstitium of patients with cryptogenic fibrosing alveolitis (CFA). Clin Exp Immunol 1995;101:436-41.

15 Furvie $H$, Yamasaki H, Suga M, et al. Altered accessory cell function of alveolar macrophages: a possible mechanism for induction of Th2 secretory profile in idiopathic pulmonary fibrosis. Eur Respir J 1997;10:787-94.

16 Wallace WA, Howie SE. Immunoreactive interleukin 4 and interferon-gamma expression by type II alveolar epithelial cells in interstitial lung disease. J Pathol 1999; 187:475-80

17 Majumdar S, Li D, Ansari T, et al. Different cytokine profiles in cryptogenic fibrosing alveolitis and fibrosing alveolitis associated with systemic sclerosis: a quantitative study of open lung biopsies. Eur Respir J 1999;14:251-7.

18 Postlethwaite AE, Holness MA, Katai H, et al. Human fibroblasts synthesize elevated levels of extracellular matrix proteins in response to interleukin-4. J Clin Invest 1992;90:1479-85.

19 Gharaee-Kermani M, Denholm EM, Phan SH. Costimulation of fibroblast collagen and transforming growth factor betal gene expression by monocyte chemoattractant protein-1 via specific receptors. J Biol Chem 1996:271:17779-84.

20 Hogaboam CM, Bone-Larson CL, Lipinski S, et al. Differential monocyte chemoattractant protein-1 and chemokine receptor 2 expression by murine lung fibroblasts derived from Th1 - and Th2-type pulmonary granuloma models. J Immunol 1999; 163:2193-201.

21 Makhluf HA, Stepniakowska J, Hoffman S, et al. IL-4 upregulates tenascin synthesis in scleroderma and healthy skin fibroblasts. J Invest Dermatol 1996; 107:856-9.

22 Postlethwaite AE, Seyer JM. Fibroblast chemotaxis induction by human recombinant interleukin-4. Identification by synthetic peptide analysis of two chemotactic domains residing in amino acid sequences 70-88 and 89-122. J Clin Invest 1991;87:2147-52.

23 Zhu Z, Homer RJ, Wang Z, et al. Pulmonary expression of interleukin-13 causes inflammation, mucus hypersecretion, subepithelial fibrosis, physiologic abnormalities, and eotaxin production. J Clin Invest 1999;103:779-88.

24 Lee CG, Homer RJ, Zhu Z, et al. Interleukin-13 induces tissue fibrosis by selectively stimulating and activating transforming growth factor beta(1). J Exp Med 2001; 194:809-21.

25 Zhu Z, Ma B, Zheng T, et al. IL-13-induced chemokine responses in the lung: role of CCR2 in the pathogenesis of IL-13-induced inflammation and remodeling. J Immunol 2002;168:2953-62.

26 Blease K, Jakubzick C, Westwick J, et al. Therapeutic effect of IL-13 immunoneutralization during chronic experimental fungal asthma. J Immunol 2001;166:5219-24

27 Blease K, Jakubzick C, Schuh JM, et al. IL-13 fusion cytotoxin ameliorates chronic fungal-induced allergic airway disease in mice. J Immunol 2001; 167:6583-92

28 Doucet C, Brouty-Boye D, Pottin-Clemenceau C, et al. IL-4 and IL-13 specifically increase adhesion molecule and inflammatory cytokine expression in human lung fibroblasts. Int Immunol 1998;10:1421-33.

29 Oriente A, Fedarko NS, Pacocha SE, et al. Interleukin- 13 modulates collagen homeostasis in human skin and keloid fibroblasts. J Pharmacol Exp Ther 2000;292:988-94.
30 Richter A, Puddicombe SM, Lordan JL, et al. The contribution of interleukin (IL)4 and IL-13 to the epithelial-mesenchymal trophic unit in asthma. Am J Respir Cell Mol Biol 2001;25:385-91.

31 Lee JH, Kaminski N, Dolganov G, et al. Interleukin-13 induces dramatically different transcriptional programs in three human airway cell types. Am J Respir Cell Mol Biol 2001;25:474-85.

32 Homer RJ, Zheng T, Chupp G, et al. Pulmonary type II cell hypertrophy and pulmonary lipoproteinosis are features of chronic IL-13 exposure. Am J Physiol Lung Cell Mol Physiol 2002;283:L52-9.

33 Hancock A, Armstrong L, Gama R, et al. Production of interleukin 13 by alveolar macrophages from normal and fibrotic lung. Am J Respir Cell Mol Biol 1998; 18:60-5.

34 Doucet C, Brouty-Boye D, Pottin-Clemenceau C, et al. Interleukin (IL) 4 and IL13 act on human lung fibroblasts. Implication in asthma. J Clin Invest 1998; 101:2129-39.

35 Murata T, Obiri NI, Debinski W, et al. Structure of IL-13 receptor: analysis of subunit composition in cancer and immune cells. Biochem Biophys Res Commun 1997:238:90-4

36 Murata T, Obiri NI, Puri RK. Structure of and signal transduction through interleukin- 4 and interleukin-13 receptors [review]. Int J Mol Med 1998; 1:551-7.

37 Obiri NI, Leland P, Murata T, et al. The IL-13 receptor structure differs on various cell types and may share more than one component with IL- 4 receptor. $\mathrm{J}$ Immunol 1997; 158:756-64.

38 Kawakami K, Taguchi J, Murata T, et al. The interleukin-13 receptor alpha2 chain: an essential component for binding and internalization but not for interleukin-13-induced signal transduction through the STAT6 pathway. Blood 2001; 97:2673-9.

39 Debinski W, Miner R, Leland P, et al. Receptor for interleukin (IL) 13 does not interact with IL4 but receptor for IL4 interacts with IL13 on human glioma cells. J Biol Chem 1996;271:22428-33.

40 Kazerooni EA, Martinez FJ, Flint A et al. Thin-section CT obtained at 10-mm increments versus limited three-level thin-section CT for idiopathic pulmonary fibrosis: correlation with pathologic scoring. AJR Am J Roentgenol 1997; 169:977-83.

41 Flaherty KR, Travis WD, Colby TV, et al. Histopathologic variability in usual and nonspecific interstitial pneumonias. Am J Respir Crit Care Med $2001 ; 164: 1722-7$

42 Flaherty KR, Colby TV, Travis WD, et al. Fibroblastic foci in usual interstitial pneumonia: idiopathic versus collagen vascular disease. Am J Respir Crit Care Med 2003;167:1410-15.

43 Hunninghake GW, Zimmerman MB, Schwartz DA, et al. Utility of a lung biopsy for the diagnosis of idiopathic pulmonary fibrosis. Am J Respir Crit Care Med 2001;164:193-6.

44 Moller DR, Forman JD, Liu MC, et al. Enhanced expression of IL-12 associated with Th1 cytokine profiles in active pulmonary sarcoidosis. J Immunol 1996;156:4952-60.

45 Smith DR, Kunkel SL, Standiford TJ, et al. Increased interleukin-1 receptor antagonist in idiopathic pulmonary fibrosis. A compartmental analysis. Am J Respir Crit Care Med 1995:151:1965-73.

46 Gordon S. Alternative activation of macrophages. Nat Rev Immunol 2003;3:23-35

47 Emura $M$, Nagai S, Takeuchi $M$, et al. In vitro production of $B$ cell growth factor and B cell differentiation factor by peripheral blood mononuclear cells and bronchoalveolar lavage $T$ lymphocytes from patients with idiopathic pulmonary fibrosis. Clin Exp Immunol 1990;82:133-9.

48 Nicholson AG. Classification of idiopathic interstitial pneumonias: making sense of the alphabet soup. Histopathology 2002;41:381-91

49 Nicholson AG, Fulford LG, Colby TV, et al. The relationship between individual histologic features and disease progression in idiopathic pulmonary fibrosis. Am J Respir Crit Care Med 2002;166:173-7.

50 Sadikot RT, Johnson J, Lloyd JE, et al. Respiratory bronchiolitis associated with severe dyspnea, exertional hypoxemia, and clubbing. Chest 2000;117:282-5.

51 Ziesche R, Hofbaver E, Wittmann K, et al. A preliminary study of long-term treatment with interferon gamma- $1 \mathrm{~b}$ and low-dose prednisone in patients with idiopathic pulmonary fibrosis. N Engl J Med 1999;341:1264-9.

52 Chiaramonte MG, Donaldson DD, Cheever AW, et al. An IL-13 inhibitor blocks the development of hepatic fibrosis during a T-helper type 2-dominated inflammatory response. J Clin Invest 1999;104:777-85.

53 Jakubzick C, Kunkel SL, Joshi BH, et al. Interleukin-13 fusion cytotoxin arrests Schistosoma mansoni egg-induced pulmonary granuloma formation in mice. Am J Pathol 2002;161:1283-97. 